\title{
Theoretical Assessment on Hetero-coordination of Alloys Silver-Antimony at Molten State
}

\author{
${ }^{a, b}$ Narayan Panthi, ${ }^{a, c}$ Indra Bahadur Bhandari, ${ }^{a}$ Ishwar Koirala* \\ ${ }^{a}$ Central Department of Physics, Tribhuvan University, Kirtipur, Nepal \\ bepartment of Physics, Patan Multiple Campus, Tribhuvan University, Nepal \\ ${ }^{\mathrm{c}}$ Department of Applied Sciences, Institute of Engineering,Tribhuvan University, Nepal \\ *Corresponding email: iswar.koirala@cdp.tu.edu.np
}

\begin{abstract}
The thermodynamic and structural properties of binary alloy Ag- Sb at temperature $1250 \mathrm{~K}$ have been reported theoretically using quasi lattice model. The interchange energy has been considered a function of a temperature and thus various thermodynamic quantities are calculated at elevated temperature. The theoretical values of free energy of mixing, heat of mixing, entropy of mixing and chemical activity are reasonable agreement with experimental values in all concentrations of antimony from 0.1 to 0.9 . The theoretical analysis tells that the alloy shows both ordering nature in Ag rich end and segregating nature in $\mathrm{Sb}$ rich end .The study reveals that the properties of alloy are asymmetric around

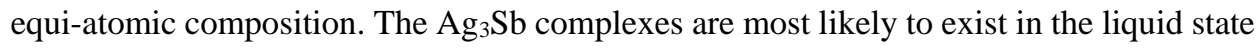
and are moderately interacting.
\end{abstract}

\begin{tabular}{l}
\multicolumn{1}{c}{ Article Info } \\
\hline Article history: \\
Received date: 07 November 2019 \\
Accepted date: 04 May 2020 \\
\hline Keywords: \\
Thermodynamic properties \\
Quasi-Lattic model 2 \\
Segregation
\end{tabular}

\section{Introduction}

The study of alloying behaviour is an important field in metallurgical science. The alloys may be of binary, ternary, quaternary and so on. Here our concern is only on the binary alloy. The binary alloy is a type of product formed by combination of two elements (at least one metal) in liquid state. Different properties of binary alloys are directly concerned with concentration of constituent elements.The alloys show symmetric or asymmetric behaviour in thermodynamic and structural properties with respect to concentration (Hultgren et al., 1973). Most of the used industrial and commercial materials are solid alloys. So they have deep utilization in solid state. The properties of initial melt play important role in the formation of alloys. Thus determination of different properties of the alloys in the liquid state are important to study the alloying behaviour in metallurgical science as well as knowledge of various properties of alloys at liquid state is important for the production of new materials required for high temperature application. Demand of different and new alloys in metallurgical science is increasing day by day and hence scientists are trying to find different alloys by mixing different elements at different compositions.

Silver is very soft, ductile and malleable metal. The combination of silver with different elements helps to improve hardness and wear-resistance of deposits and higher stability tarnishing antifriction characteristics. There has been growing tendency in calculating the properties of silver-antimony alloys. It has been used as electroplating deposition due to its increased hardness and wear-resistance which, finally aims to the use of smaller plate thickness and consequent saving of expensive materials (Singh et al., 2010).

Different theoreticians are working with various theoretical models to understand the alloying behaviour of compound forming binary alloys. In the present work, one of the Silver alloys Ag-Sb is studied theoretically to determine certain properties at $1250 \mathrm{~K}$ assuming $\mathrm{Ag}_{\mathrm{x}} \mathrm{Sb}_{\mathrm{y}}(\mathrm{x}=3, \mathrm{y}=1)$ complex in melt by using Quasi lattice model. Thermodynamic properties such as free energy of mixing, heat of mixing and entropy of mixing, chemical activity provide the knowledge on the interaction, stability and bonding strength among the constituent atoms whereas information on 
the structural ordering of atoms in binary alloys in the liquid state is provided by the quantitative analysis of microscopic functions, the concentration fluctuations in the long wavelength limit $(\operatorname{Scc}(0))$, short-range order parameter $(\alpha 1)$ and diffusivity $\left(\mathrm{D}_{\mathrm{M}} / \mathrm{D}_{\mathrm{id}}\right)$ (Koirala et al., 2014; Jha et al., 2015; Koirala et al., 2018). Scc(0) represents the nature of ordering of the atoms , $\alpha 1$ quantifies the degree of ordering and diffusion coefficient ratio $\left(\mathrm{D}_{\mathrm{M}} / \mathrm{D}_{\mathrm{id}}\right)$ gives the structural behaviour of the alloy .

The organization of this paper is as follows. In Section 2, the expressions required for the calculation are presented. In Section 3, result and general discussion of the alloy $\mathrm{Ag}-\mathrm{Sb}$ are presented. Finally the conclusions are given in Section 4.

\section{Theoretical Formulation}

Let a binary alloy contains in all $N$ atoms with $\mathrm{N}_{\mathrm{A}}$ and $\mathrm{N}_{\mathrm{B}}$ atoms of $\mathrm{A}$ and $\mathrm{B}$ elements respectively, $k_{\mathrm{B}}$ is Boltzmann constant, $T$ is absolute temperature. Then the model considers the existence of chemical complexes $\mathrm{A}_{x} \mathrm{~B}_{\mathrm{y}}$ where, $\mathrm{A}$ and $\mathrm{B}$ are the constituent species of the alloy and $x$ and $y$ are the small integers. $x \mathrm{~A}+y \mathrm{~B}=A_{x} B_{y}$

With this consideration, grand partition function (Guggenheim, 1952) in terms of configurational energy ' $\mathrm{E}$ ' is expressed as

$\Xi=\sum_{E} q_{A}^{N_{A}}(T) q_{B}^{N_{B}}(T) \exp \left[\left(\mu_{A} N_{A}+\mu_{B} N_{B}-E\right) / k_{\mathrm{B}} \mathrm{T}\right]$

Where $q_{i}(T)$ and $\mu_{i}$ are atomic partition function and chemical potential of the $i^{\text {th }}(\mathrm{i}=\mathrm{A}, \mathrm{B})$ species. By using above relation we find following expressions for the determination of different properties. With this consideration the excess free energy of mixing becomes

$G_{M}^{X S}=N K_{B} T \int_{0}^{c} \gamma d c$

Where $\gamma$ is ratio of the activity coefficient of atom A to $\mathrm{B} ; \mathrm{C}$ is the concentration of atom $\mathrm{A}$

The solution of $\mathrm{eq}^{\mathrm{n}}$. (2) leads to

$G_{M}^{X S}=N\left[\phi \omega+\phi_{A B} \omega_{A B}+\phi_{A A} \omega_{A A}+\phi_{B B} \omega_{B B}\right]$

Where $\omega$ 's are the ordering energies; and $\Phi=\mathrm{c}(1$ c) and $\Phi \mathrm{ij}$ 's $(\mathrm{i}, \mathrm{j}=\mathrm{A}, \mathrm{B})$ are the simple polynomials in c depending on the values of $x$ and $y$.

For $\mathrm{A}=\mathrm{Ag}, \mathrm{B}=\mathrm{Sb}, x=3$ and $\mathrm{y}=1$, the values of $\Phi_{\mathrm{ij}}$ 's (Bhatia \& Singh, 1982) are found to be

$\Phi_{\mathrm{AB}}(\mathrm{c})=\frac{1}{5} c+\frac{2}{3} c^{3}-c^{4}-\frac{1}{5} c^{5}+\frac{1}{3} c^{6}$
$\Phi_{\mathrm{AA}}(\mathrm{c})=-\frac{3}{20} c+\frac{2}{3} c^{3}-\frac{3}{4} c^{4}+\frac{2 c^{5}}{5}-\frac{1}{6} c^{6}$

$\Phi_{B B}(c)=0$

The Free energy of mixing for complex forming

$$
\begin{aligned}
& \begin{aligned}
G_{\mathrm{M}} & =G_{\mathrm{M}}^{\mathrm{XS}}+N k_{\mathrm{B}} T[c \ln c+(1-c) \ln (1-c)] \\
& =R T\left[\Phi \frac{\omega}{k_{\mathrm{B}} T}+\Phi_{\mathrm{AB}} \frac{\Delta \omega_{\mathrm{AB}}}{k_{\mathrm{B}} T}+\Phi_{\mathrm{AA}} \frac{\Delta \omega_{\mathrm{AA}}}{k_{\mathrm{B}} T}+\Phi_{\mathrm{BB}} \frac{\Delta \omega_{\mathrm{BB}}}{k_{\mathrm{B}} T}+\right.
\end{aligned} \\
& c \ln c+(1-c) \ln (1-c)]
\end{aligned}
$$

The heat of mixing is found out by using standard thermodynamic relation:

$$
\begin{aligned}
\frac{H_{M}}{R T} & =\frac{G_{\mathrm{M}}}{R T}-\left[\frac{1}{R} \frac{d G_{\mathrm{M}}}{d T}\right] \mathrm{c}, \mathrm{N}, \mathrm{P} \\
& =\Phi\left[\frac{\omega}{k_{\mathrm{B}} T}-\frac{1}{k_{\mathrm{B}}} \frac{d \omega}{d T}\right]+\Phi_{\mathrm{AB}}\left[\frac{\Delta \omega_{\mathrm{AB}}}{k_{\mathrm{B}} T}-\frac{1}{k_{\mathrm{B}}} \frac{\mathrm{d} \Delta \omega_{\mathrm{AB}}}{d T}\right]+ \\
\Phi_{\mathrm{AA}} & {\left[\frac{\Delta \omega_{\mathrm{AA}}}{k_{\mathrm{B}} T}-\frac{1}{k_{\mathrm{B}}} \frac{\mathrm{d} \Delta \omega_{\mathrm{AA}}}{d T}\right]+\Phi_{\mathrm{BB}}\left[\frac{\Delta \omega_{\mathrm{BB}}}{k_{\mathrm{B}} T}-\right.} \\
& \left.\frac{1}{k_{\mathrm{B}}} \frac{\mathrm{d} \Delta \omega_{\mathrm{BB}}}{d T}\right]
\end{aligned}
$$

The standard thermodynamic relation for entropy of mixing is

$\frac{S_{\mathrm{M}}}{R}=\frac{H_{M}}{R T}-\frac{G_{\mathrm{M}}}{R T}$

The activity of the constituent elements in the alloys is determined from standard thermodynamic relation $\mathrm{RT} \ln a_{i}(\mathrm{i}=\mathrm{A}, \mathrm{B})=\mathrm{GM}+\left(1-c_{i}\right)\left[\frac{\partial G_{M}}{\partial C_{i}}\right]_{T, P, N}$

By solving $\mathrm{eq}^{\mathrm{ns}}(4)$ and (7), the theoretical values activities of each component are given as follows.

$$
\begin{aligned}
& \ln a_{A}=\frac{G_{M}}{R T}+\frac{1-C}{K_{B} T}\left[(1-2 \mathrm{c}) \omega+\Phi_{A B}^{\prime} \Delta \omega_{A B}+\right. \\
& \left.\left.\Phi_{A A}^{\prime} \Delta \omega_{A A}+\Phi_{B B}^{\prime} \Delta \omega_{B B}+\ln \frac{C}{1-C}\right)\right] \\
& \ln a_{B}=\frac{G_{M}}{R T}+\frac{C}{K_{B} T}\left[(1-2 \mathrm{c}) \omega+\Phi_{A B}^{\prime} \Delta \omega_{A B}+\right. \\
& \left.\Phi_{A A}^{\prime} \Delta \omega_{A A}+\Phi_{B B}^{\prime} \Delta \omega_{B B}+\ln \left(\frac{C}{1-C}\right)\right]
\end{aligned}
$$

Where, $\Phi_{A B}^{\prime}, \Phi_{A A}^{\prime}$ and $\Phi_{B B}^{\prime}$ are concentration derivatives of $\Phi_{\mathrm{AB}}, \Phi_{\mathrm{AA}}$ and $\Phi_{\mathrm{BB}}$ respectively.

The concentration fluctuation in long wavelength limit (Bhatia \& Thornton,1970) for alloy is derived from standard relation

$$
\begin{aligned}
S_{\mathrm{cc}}(0) & =R T\left[\frac{\partial^{2} G_{\mathrm{M}}}{\partial c^{2}}\right]_{T, P, N}^{-1} \\
& =c_{2} a_{1}\left[\frac{\partial a_{1}}{\partial C_{1}}\right]_{T, P,}^{-1}=c_{2} a_{2}\left[\frac{\partial a_{2}}{\partial C_{2}}\right]_{T, P}^{-1}
\end{aligned}
$$

Where $\mathrm{c}_{1}(=\mathrm{c})$ and $\mathrm{c}_{2}(=1-\mathrm{c})$ are concentrations and $a_{1}$ and $a_{2}$ are observed activities of elements $\mathrm{A}$ and $\mathrm{B}$ respectively.

Solving eq ${ }^{\text {ns }}$ (4) and 10(a), the theoretical value of $\operatorname{Scc}(0)$ is found as follows, 
$\operatorname{Scc}(0)=\frac{c(1-c)}{1+c(1-c)\left\{-2 \frac{\omega}{k_{\mathrm{B}} T}+\phi_{A B}^{\prime \prime} \frac{\Delta \omega_{\mathrm{AB}}}{k_{\mathrm{B}} T}+\phi_{A A}^{\prime \prime} \frac{\Delta \omega_{\mathrm{AA}}}{k_{\mathrm{B}} T}+\phi_{B B}^{\prime \prime} \frac{\Delta \omega_{\mathrm{BB}}}{k_{\mathrm{B}} T}\right\}}$

Where $\phi_{i, j}^{\prime \prime}=\frac{\partial^{2} \phi_{i, j}}{\partial c^{2}}(\mathrm{i}, \mathrm{j}=\mathrm{A}, \mathrm{B})$

The Warren-Cowley short order parameter (Warren, 1969; Cowle, 1950) is related with concentration fluctuation in long wavelength limit as $\alpha 1=\frac{S-1}{S(Z-1)+1}$, where $\mathrm{S}=\frac{S_{c c(0)}}{S_{c c(0)}^{i d}}$

$S_{c c}^{i d}(0)=c(1-c)$

The mixing behavior of the alloy forming molten can also be studied at the microscopic level in terms of diffusion coefficient ratio which is related to concentration fluctuation in long wave length limit as, $\frac{D_{M}}{D_{i d}}=\frac{S_{c c(0)}^{i d}}{S_{c c(o)}}$

Where $D_{i d}$ is the intrinsic diffusion coefficient for an ideal mixture and $D_{M}$ is chemical or mutual diffusion coefficient.

$\mathrm{D}_{\mathrm{M}}$ is given as (Darken \& Gurrey, 1953; Singh et al., 2014)

$D_{M}=D_{i d} C_{i} \frac{d \ln a_{i}}{d C_{i}}$

\section{Results and Discussion}

\subsection{Thermodynamic properties}

\section{Free Energy of Mixing}

The energy parameters used for free energy of mixing for $\mathrm{Ag}-\mathrm{Sb}$ liquid alloy is determined by successive approximation method. The parameters are determined by using Eqn. (4) with experimental value of $G_{M}$ in the concentration range from 0.1 to 0.9 (Hultgren et al.,1973). The best fit values of the parameters are

$\frac{\omega}{k_{\mathrm{B}} T}=-3.97, \frac{\Delta \omega_{\mathrm{AB}}}{k_{\mathrm{B}} T}=4.38, \frac{\Delta \omega_{\mathrm{AA}}}{k_{\mathrm{B}} T}=-2.68$

Eqn. (4) is used to compute the free energy of mixing $\left(\mathrm{G}_{\mathrm{M}} / \mathrm{RT}\right)$ for $\mathrm{Ag}-\mathrm{Sb}$ liquid alloy. The plot of free energy of mixing verses concentration of antimony is shown in Fig. 1. The computed and experimental values of $\mathrm{G}_{\mathrm{M}} / \mathrm{RT}$ are in good agreement. The theoretical value of free energy of mixing is minimum i.e. $-1.2 \mathrm{RT}$ at $\mathrm{C}_{\mathrm{Sb}}=0.4$. The theoretical calculation of free energy of mixing shows that at liquid state, the alloy $\mathrm{Ag}-\mathrm{Sb}$ is moderately interacting and hence the tendency of compound formation is not so strong.

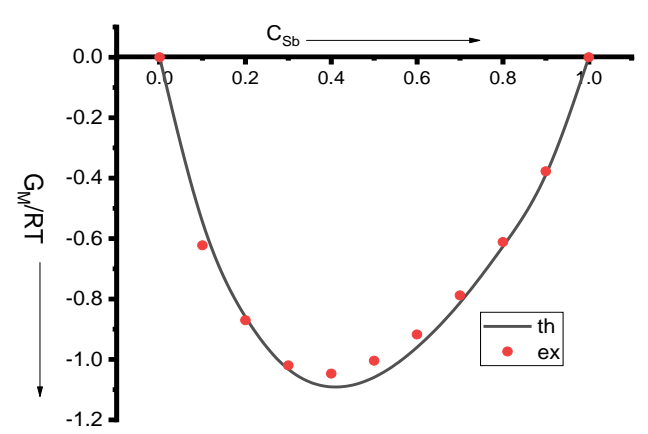

Fig. 1: Free energy of mixing $\left(\mathrm{G}_{\mathrm{M}} / \mathrm{RT}\right)$ vs concentration of antimony $\left(\mathrm{C}_{\mathrm{Sb}}\right)$ in liquid alloy at $1250 \mathrm{~K}$

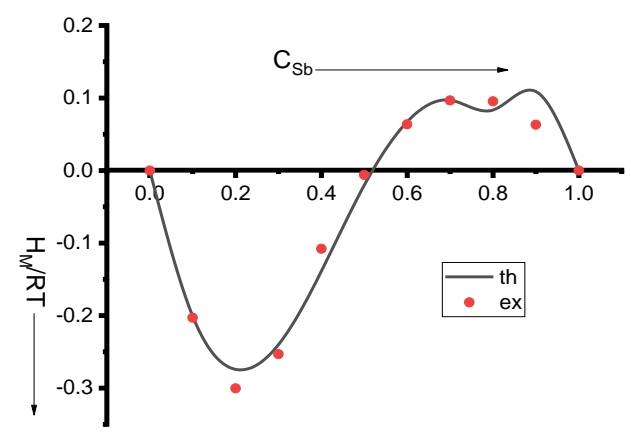

Fig. 2: Heat of mixing $\left(\mathrm{H}_{\mathrm{M}} / \mathrm{RT}\right)$ vs concentration of antimony $\left(\mathrm{C}_{\mathrm{Sb}}\right)$ in liquid alloy at $1250 \mathrm{~K}$

\section{Heat of Mixing}

For the theoretical determination of heat of mixing temperature derivatives of interaction parameters are done. The observed values of $H_{M}$ (Hultgren et al., 1973) are used by successive approximation method. The best fit values of parameters are

$\frac{1}{k_{\mathrm{B}}} \frac{d \omega}{d T}=2.28, \frac{1}{k_{\mathrm{B}}} \frac{\mathrm{d} \Delta \omega_{\mathrm{AB}}}{d T}=-7.89, \frac{1}{k_{\mathrm{B}}} \frac{\mathrm{d} \Delta \omega_{\mathrm{AA}}}{d T}=-4.32$

Eqn. (5) is used to compute the heat of mixing $\left(\mathrm{H}_{\mathrm{M}} / \mathrm{RT}\right)$ for Ag-Sb alloy. The plot of heat of mixing verses concentration of antimony is shown in Fig. 2. It is found from the analysis that the heat of mixing is negative in antimony rich region whereas it is positive in the silver rich region. The observed S-shaped nature of heat of mixing verses concentration curve is well explained by the theory. The computed and experimental values of $\mathrm{H}_{\mathrm{M}} / \mathrm{RT}$ are in reasonable agreement with some discrepancies.

\section{Entropy of Mixing}

Using equation (6) along with equations (4) and (5), the entropy of mixing $\left(\mathrm{S}_{\mathrm{M}}\right)$ is computed. For theoretical calculation same energy parameters eq ${ }^{\mathrm{n}}(17)$ are used.

The plot of entropy of mixing $\left(\mathrm{S}_{\mathrm{M}} / \mathrm{R}\right)$ verses concentration of antimony is shown in Fig. 3 for the both theoretical and observed values. From figure, it is 
observed that theoretical values are in good agreement with observed values.

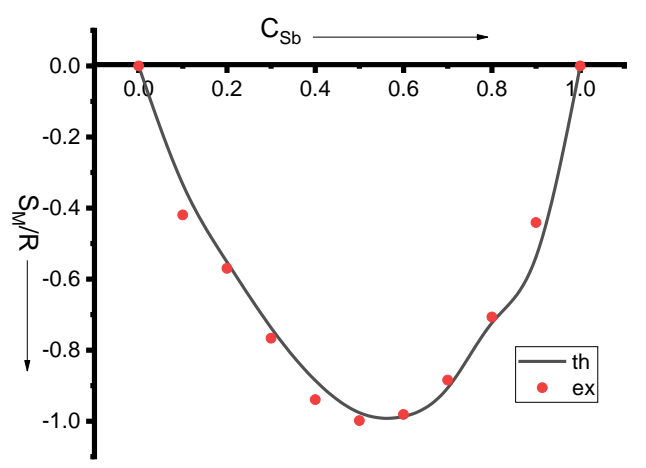

Fig. 3: Entropy of mixing $\left(S_{M} / R\right)$ vs concentration of antimony $\left(\mathrm{C}_{\mathrm{Sb}}\right)$ in liquid alloy at $1250 \mathrm{~K}$

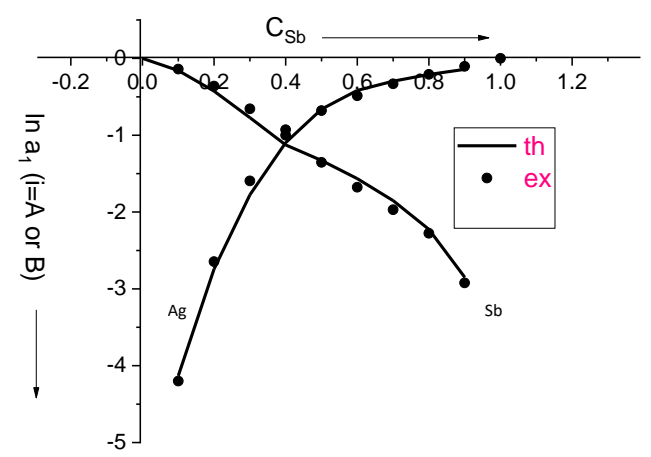

Fig. 4: Chemical activity vs concentration of antimony $\left(\mathrm{C}_{\mathrm{Sb}}\right)$ in liquid alloy at $1250 \mathrm{~K}$

\section{Chemical activity}

The deviation from ideal behavior of alloys can be explained by chemical activitities. The eq ${ }^{\text {ns }}(8)$ and (9) are used for theoretical calculation of chemical activity of constituent elements of alloy Ag-Sb. Fig. 4 shows the observed and theoretical values of chemical activity of the alloy.There is good aggrement between experimental and thheoretical values of activity of $\mathrm{Ag}$ and $\mathrm{Sb}$ in $\mathrm{Ag}-\mathrm{Sb}$ alloys at $1250 \mathrm{~K}$ at all concentrations of $\mathrm{Sb}$.

\subsection{Structural properties}

\section{Concentration fluctuation in long wave length limit}

One of the important function for the study of nature of atomic order of the binary liquid is considered as the Concentration fluctuations in the long-wavelength $\operatorname{limit}(\operatorname{Scc}(0))$ because it removes difficulties in diffraction experiment(Bhatia \& Thornton, 1970). For given concentration if $\operatorname{Scc}(0)>S^{\text {id }} \operatorname{cc}(0)$, the alloy is expected to have tendency of segregating while if $\operatorname{Scc}(0)<\mathrm{S}^{\mathrm{id}} \operatorname{cc}(0)$, the expected nature is complex formation.The experimental and theoretical values of $\operatorname{Scc}(0)$ at different concentrations of element antimony are obtained from eq ${ }^{\mathrm{ns}}(10)$ and (11) respectively. The plot of experimental and theoretical along with ideal values of $\operatorname{Scc}(0)$ verses concentration is shown in Fig.5.

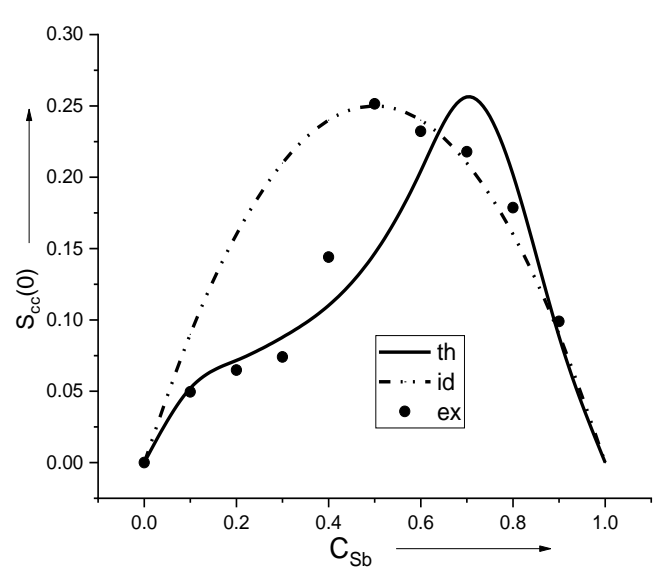

Fig. 5: Concentration fluctuation in long wavelength $\operatorname{limit}\left(\mathrm{S}_{\mathrm{cc}}(0)\right)$ vs concentration of antimony $\left(\mathrm{C}_{\mathrm{Sb}}\right)$ in liquid alloy at $1250 \mathrm{~K}$

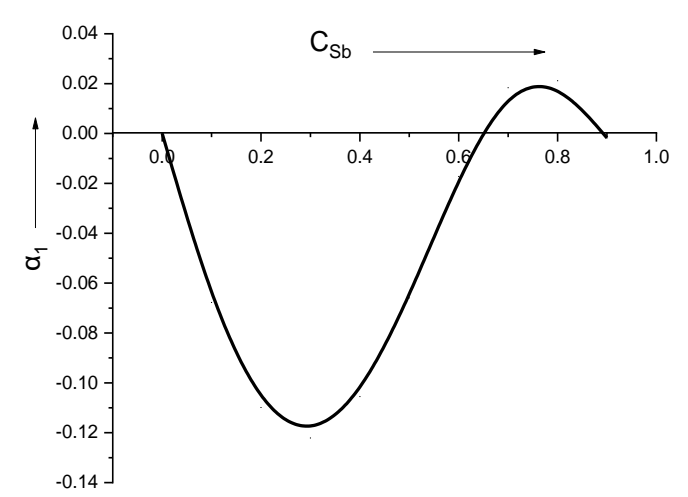

Fig. 6: Waren-Cowley short range order parameter vs concentration of antimony $\left(\mathrm{C}_{\mathrm{Sb}}\right)$ in liquid alloy at $1250 \mathrm{~K}$

\section{Warren- Cowley short range order parameter}

The Warren-Cowley short-range orderparameter $\left(\alpha_{1}\right)$ is one of the useful parameter to quantify thedegree of chemical order in the alloy melt. It provides information of the local arrangement of the atoms in the molten alloys. For the equi-atomic composition, the Warren-Cowley short range orderparameter is found to be $-1 \leq \alpha_{1} \leq 1$. Negative values of $\alpha_{1}$ indicate ordering nature in the melt, which is complete if $\alpha_{1}=-1$. On the other hand, positive values of $\alpha_{1}$ indicate segregating nature, which is completeonly if $\alpha_{1}=1$. But $\alpha_{1}=0$, indicates the random distribution of the atoms in the mixture. The $\operatorname{Scc}(0)$ and $\alpha_{1}$ have been computed as a function of concentration of Sb using Eqns. (12) and (13) respectively. The plot of theoretical value of $\alpha_{1}$ verses concentration of $\mathrm{Sb}$ is shown in Fig. 6.

The Fig. 6 also shows that $\alpha_{1}$ is between concentrations 0 to 0.62 of $\mathrm{Sb}$ which is the indication of unlike atoms pairing between constituent atoms. 


\subsection{Diffusivity}

In terms of diffusion coefficient ratio, mixing behavior of liquid alloys can also be explained at the microscopic level. The relationship between $\operatorname{Scc}(0)$ and the diffusivity is expressed by the ratio of the mutual and self-diffusion coefficients $\left(D_{M} / D_{i d}\right)$ which indicates the mixing behavior of the alloys, i.e., the tendency for heterocoordination $\left(\mathrm{D}_{\mathrm{M}} / \mathrm{D}_{\mathrm{id}}>1\right)$ or homocoordination nature ofatoms $\left(\mathrm{D}_{\mathrm{M}} / \mathrm{D}_{\mathrm{id}}<1\right)$ or ideal $\operatorname{mixing}\left(\mathrm{D}_{\mathrm{M}} / \mathrm{D}_{\mathrm{id}}=1\right)$.

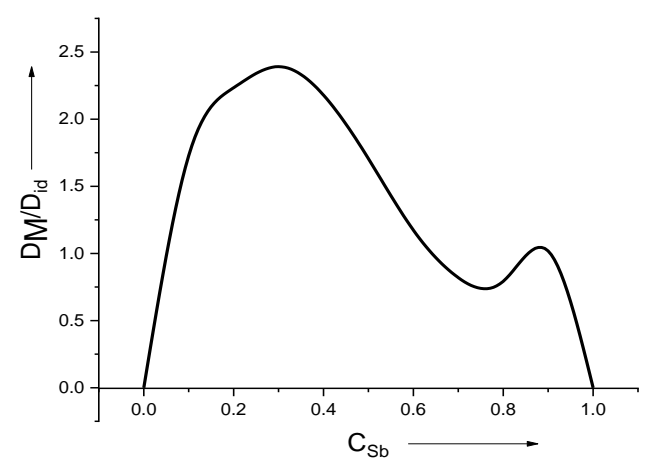

Fig. 7: Diffusion coefficient ratio vs concentration of antimony $\left(\mathrm{C}_{\mathrm{Sb}}\right)$ in liquid alloy at $1250 \mathrm{~K}$

The calculated values of $\operatorname{Scc}(0)$ are used in $\mathrm{eq}^{\mathrm{n}}$. (15) to determine the ratio of the mutual and intrinsicdiffusion coefficients $\left(D_{M} / D_{\text {id }}\right)$. Fig. 7 shows the plot of $\mathrm{D}_{\mathrm{M}} / \mathrm{D}_{\text {id }}$ against the concentration of antimony. In the figure, the value of $D_{M} / D_{\text {id }}$ is found to be less than 1 in the range of concentration, which is indicative of the phase separarion in the mixture. A maximum value of $\mathrm{D}_{\mathrm{M}} / \mathrm{Di}_{\mathrm{d}}=2.39$ for concentration of $\mathrm{Sb}=0.3$ confirms tendency for chemical ordering, observed by the $\operatorname{Scc}(0)$ and chemical short range orded parameter.

\section{Conclusions}

The thermodynamic properties of $\mathrm{Ag}-\mathrm{Sb}$ alloy at melting temperature $1250 \mathrm{~K}$ are examined on the basis of Quasi- lattice model. The model successively explains concentration dependent nature of the alloys by assuming $\mathrm{Ag}_{3} \mathrm{Sb}$ complex in melt. From analysis it is justified that the alloy is moderately interacting in nature and hence it doesn't have strong tendency of compound formation. The energy parameters depend on temperature.

\section{Acknowledgments}

The authors are grateful to Dr. N.P. Adhikari, Professor, Department of Physics, Tribhuvan University, Kathmandu and R. K. Bachchan, Lecturer,
Patan Multiple Campus, T.U. for helpful discussions on this work.

\section{Conflicts of Interest}

We wish to confirm that there are no known conflicts of interest associated with this publication and there has been no significant financial support for this work that could have influenced its outcome.

\section{Funding}

This research received no external funding.

\section{References}

Bhatia, A. B., \& Singh, R. N. (1982). Thermodynamic properties of compound forming molten Alloys in a weak interaction approximation. Physics and Chemistry of Liquids an International Journal, 11(4), 343-351.

Bhatia, A. B., \& Thornton, D. E. (1970). Structural aspects of the electrical resistivity of binary alloys. Physical Review B, 2(8), 3004.

Cowley, J. M. F. (1950). An approximate theory of order in alloys. Physical Review, 77(5),669.

Darken, L. S., \& Gurry, R. W. (1953). Physical Chemistry of Metals. Mc Granw-Hill Book Company. Inc. New York Toronto London, 582.

Guggenheim, E. A. (1952). Mixture. Oxford University press. London

Hultgren, R., Desai, P. D., Hawkins, D. T., Gleiser, M., \& Kelley, K. K., (1973). Selected Values of Thermodynamic Properties of Binary Alloys. American Society for Metals, Metals Park.

Jha, I. S., Koirala, I., Singh, B. P., \& Adhikari. D. (2014). Concentration dependence thermodynamic, transport and surface properties in $\mathrm{Ag}-\mathrm{Cu}$ liquid alloys. Applied Physics A, 116(3), 1517-1523.

Koirala, I., Singh, B. P., \& Jha, I. S. (2015). Theoretical Investigation of Mixing Behavior of Al$\mathrm{Fe}$ Alloys in Molten Stage. African review of physics, 10, 329-335.

Koirala, R. P., Koirala, I., \& Adhikari, D. (2018). Energetics of mixing and transport phenomena in $\mathrm{Cd}-\mathrm{X}(\mathrm{X}=\mathrm{Pb}, \mathrm{Sn})$ melts. Bibechana, 15, 113-120.

Singh, B. P., Adhikari, D., \& Jha, I. S. (2010). Concentration dependence of the structure and thermodynamic properties of silver-antimony 
alloys. Journal of Non-Crystalline Solids, 356(33$34), 1730-1734$.

Singh, B. P., Koirala, I., Jha, I. S., \& Adhikari, D. (2014). The segregating nature of $\mathrm{Cd}-\mathrm{Pb}$ liquid binary alloys. Physics and Chemistry of liquids, 52(4), 457-470.

Warren, B. E. (1969). X-ray Diffraction, Reading MA, Addison-Wesley.

(c) (P)

(C) 2020 by the authors. Submitted for possible open access publication under the terms and conditions of the Creative Commons Attribution (CC BY) license (http://creativecommons.org/licenses/by/4.0/).

How to cite: Panthi, N., Bhandari, I. B., \& Koirala, I. (2020). Theoretical Assessment on Hetero-coordination of Alloys Silver-Antimony at Molten State. Himalayan Journal of Science and Technology, 3-4, 68-73. 